\title{
A low-cost wireless endoscope camera: a preliminary report
}

\author{
J. M. Lazarus* (i) and M. Ncube
}

\begin{abstract}
Background: Technology currently used for surgical endoscopy was developed and is manufactured in high-income economies. The cost of this equipment makes technology transfer to resource constrained environments difficult. We aimed to design an affordable wireless endoscope to aid visualisation during rigid endoscopy and minimally invasive surgery (MIS). The initial prototype aimed to replicate a 4-mm lens used in rigid cystoscopy.

Methods: Focus was placed on using open-source resources to develop the wireless endoscope to significantly lower the cost and make the device accessible for resource-constrained settings. An off the shelf miniature single-board computer module was used because of its low cost (US\$10) and its ability to handle high-definition (720p) video. Open-source Linux software made monitor mode ("hotspot") wireless video transmission possible. A $1280 \times 720$ pixel high-definition tube camera was used to generate the video signal. Video is transmitted to a standard laptop computer for display. Bench testing included latency of wireless digital video transmission. Comparison to industry standard wired cameras was made including weight and cost. The battery life was also assessed.
\end{abstract}

Results: In comparison with industry standard cystoscope lens, wired camera, video processing unit and light source, the prototype costs substantially less. (US\$ $230 \mathrm{vs} 28$ 000). The prototype is light weight (184 g), has no cables tethering and has acceptable battery life (of over $2 \mathrm{~h}$, using a $1200 \mathrm{mAh}$ battery). The camera transmits video wirelessly in near real time with only imperceptible latency of $<200 \mathrm{~ms}$. Image quality is high definition at 30 frames per second. Colour rendering is good, and white balancing is possible. Limitations include the lack of a zoom.

Conclusion: The novel wireless endoscope camera described here offers equivalent high-definition video at a markedly reduced cost to contemporary industry wired units and could contribute to making minimally invasive surgery possible in resource-constrained environments.

\section{Background}

Conventional surgical endoscopy has for decades relied on robust and time-tested lens technology developed in the 1960s. The traditional endoscope uses multiple glass rod lenses (see Fig. 1) which were invented by Harold $\mathrm{H}$ Hopkins and fibre-optic cold light source developed by Karl Storz [1, 2]. "Operating off the monitor" became possible in the late 1970s when a video image could be transmitted to a monitor via a camera connected to the eyepiece [3]. It is testament to the revolutionary work

\footnotetext{
*Correspondence: j.lazarus@uct.ac.za
}

Division of Urology, University of Cape Town, Cape Town, South Africa of these early pioneers that in many ways endoscopy is unchanged since the 1960s.

There are, however, drawbacks to traditional endoscopy systems including:

(1) the significant weight of the endoscope, the camera and their cables,

(2) the reduced manoeuvrability afforded by the external cables and

(3) since the lens is relatively fragile it can easily be damaged requiring costly replacement.

Miniaturisation of optic and electronic components has ushered in an era where these drawbacks can potentially 


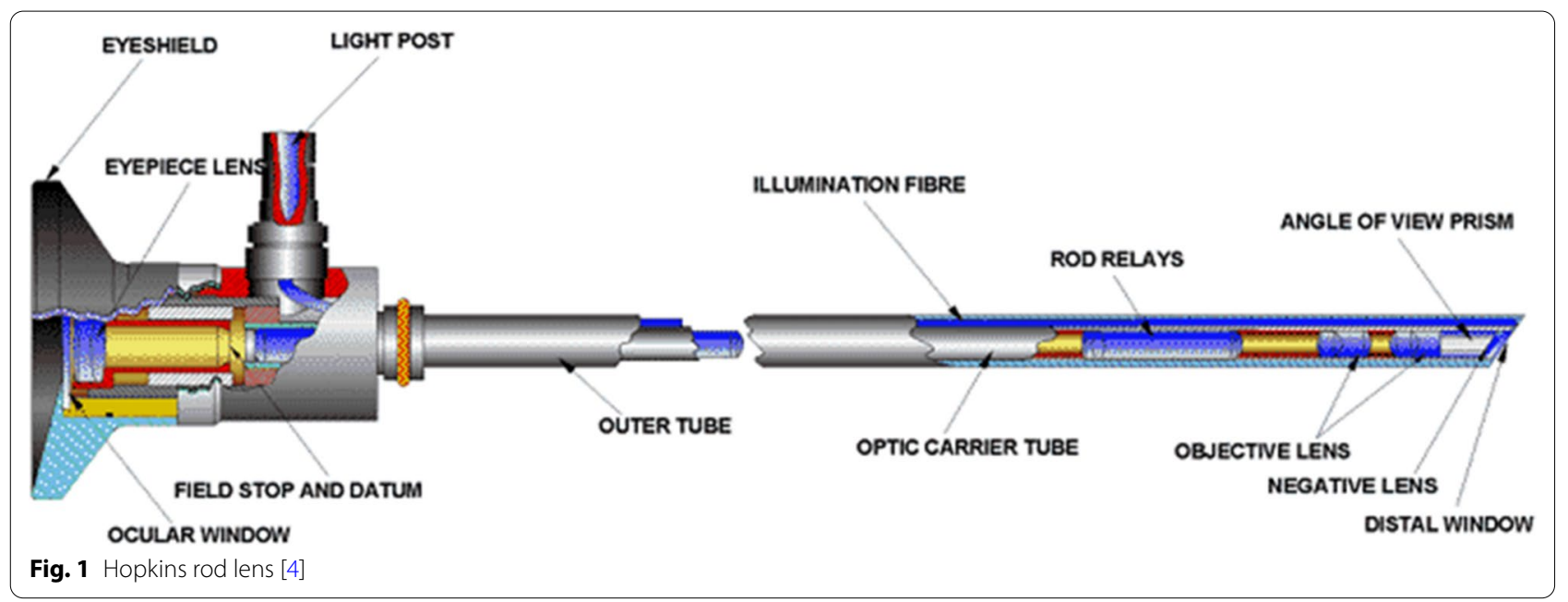

be overcome. The literature includes description of a wireless laparoscope [5], devices which replace the traditional endoscope camera with a smart phone [6], robotassisted rigid and flexible endoscopy [7] and miniature wireless "capsule" endoscopy for the specific application of gastrointestinal survey [8].

In this study, we will describe the development and bench testing of a novel wireless endoscope which aims to achieve the following:

(1) to replace the Hopkins glass rod lenses with a miniature camera,

(2) to replace fibre-optic illumination with miniature light emitting diodes (LEDs),

(3) to replace the external camera cables with wireless video transmission,

(4) and to replace power cables with incorporated rechargeable batteries.

Open surgery has not given way to minimally invasive surgery (MIS) in the developing world. One of the oftencited reasons for this is the cost of MIS equipment. MIS (whether laparoscopy or endoscopy) relies on the Hopkins rod lens. These lenses are expensive and fragile. It is postulated that the novel wireless endoscope described here holds potential to overcome the cost and technical limitations of traditional endoscopy.

\section{Methods}

We focused on using "off the shelf" components and open-source software to develop the wireless endoscope. This significantly lowered the cost with the goal to make the device accessible for resource-constrained environments. While the technology described is applicable to a variety of rigid lens applications, we attempted to replicate the $4 \mathrm{~mm}$ cystoscope lens and separately a clipon universal endoscope camera.

\subsection{Hardware systems}

A miniature single-board computer (SBC) module the Raspberry Pi Zero W (Raspberry Pi Foundation, Caldecote, UK) was used because of its size, its low cost (US\$10) and its ability to handle high-definition (720p) video.

A $3.7-\mathrm{mm}$ tube camera (model: 1001LG, Shenzhen Eastern International Corporation Limited, Shenzhen, China) was used. It delivers $1280 \times 720$ high-definition video using a $1 / 7^{\prime \prime}$ colour CMOS sensor. Lens construction allows a wide (115 degree) field of view and an extended depth of field allowing object in the range of 5 to $50 \mathrm{~mm}$ to be in focus. Connectivity is via a USB 2.0 interface with the SBC. The camera is certified IP67 waterproof and the manufacturer is ISO 13485:2016 certified for the design and manufacturer of medical endoscope cameras.

Illumination is via 6 high luminous 0603 white colour LEDs incorporated into the tube camera. The system was powered by a $1200 \mathrm{mAh}$ lithium polymer battery and incorporated into a fireproof acrylonitrile butadiene styrene enclosure.

For the clip-on wireless camera module, an $18-35 \mathrm{~mm}$ optical zoom coupler (Ouman Medical, Jiangsu Ouman Electronic Equipment Co., ltd, Jiangsu, China) and an 8-megapixel camera module (model: IMX219, Arducam) were used.

\subsection{Software systems}

Open-source Linux software was used on the SBC as follows: 
1. The SBC runs on the Raspbian Pi operating system Lite, a minimal image of Debian Buster [9].

2. The SBC Wi-Fi module is placed in monitor mode ("hotspot") using RaspAP [10]. This makes wireless video transmission possible.

3. The video signal is streamed via the UV4L module of "Video 4 Linux 2" [11].

4. Lastly, the wireless video signal is viewed on a standard computer via any internet browser. Figure 2 illustrates the entire software setup.

\subsection{Study protocol}

The following pre-clinical bench testing was performed:

(a) Latency of wireless digital video transmission was assessed. Here, the time delay inherent in the wireless transmission was assessed by photographing a mobile phone stopwatch with an accuracy of $1 \mathrm{~ms}$. Comparison could be made between the phone time and the wireless image transmitted from the SBC to a standard laptop.

(b) Weight of the wireless camera was assessed.

(c) A cost comparison with an industry standard camera, Hopkins lens, light source and video processing unit was made.

(d) Lastly, the timed battery life of the novel wireless camera was assessed while transmitting a high-definition video signal at 30 frames per second.

\section{Results}

Figure 3 illustrates the prototype wireless endoscope cameras. The novel endoscope was capable of image quality of 720 p high definition $(1280 \times 720$ pixels $)$ at 30 frames per second. It transmitted video wirelessly in near real time with only imperceptible latency of roughly $200 \mathrm{~ms}$. Figure 4 illustrates the latency on a mobile phone stopwatch.

In comparison with wired endoscopes, the prototype costs substantially less than a contemporary wired camera and video processing unit. [US\$ 230 vs 28000 (Karl Storz - Image 1, Karl Storz SE \& Co. Tuttlingen, Germany)].
The prototype is light weight ( $184 \mathrm{~g}$ ) and has no cables tethering. The battery life was over $2 \mathrm{~h}$, using a $1200 \mathrm{mAh}$ battery. White balancing was possible.

Limitations of the wireless tube camera include lack of a zoom on the camera, but it can be done via the receiving computer's internet browser. Additionally, unlike the traditional Hopkins rod-lenses, the wireless camera lacks a $30^{\circ}$ down capability. The assessment of surgeon appreciation of image quality, colour rendering, latency and other safety and efficacy aspects of the camera would require a formal pilot trial.

\section{Discussion}

This paper has outlined the development of a novel wireless endoscope camera. Traditional wired cameras using Hopkins lenses have recognised limitation. Specifically, for the developing world the cost of these technologies has retarded the growth of minimally invasive surgery. Via miniaturisation of cameras and using LED illumination, there exists the possibility that affordable endoscopy could allow growth of MIS in the developing world for the benefit of surgeons and their patients.

The application of wireless transmission in endoscopy was confined to the capsule endoscopy in the past [8]. These miniature "capsules" are ingested and provide wireless gastrointestinal imaging survey for cancer diagnosis.

Bae et al. have described a novel smartphone adaptor which elegantly allows the smartphone screen to display the endoscope image [6]. Unlike the wireless endoscope described in our study, a light source is required, and the display is limited to the phone's screen instead of a traditional larger monitor.

The application of wireless transmission in rigid endoscopy has been largely neglected. One exception is a paper by Chen et al. [5] who described the development of a wireless "electrical non-fiberoptic endoscope". They tested the wireless laparoscope in an animal model and noted that its feasibility and safety and postulated that wireless scopes may have a role in endoscopic surgery in the future.

Wireless transmission has several advantages over traditional wired cameras. We have shown that the weight of the cameras is markedly reduced. Wireless cameras lack tethering cables which limit manoeuvrability and reduce ergonomics.

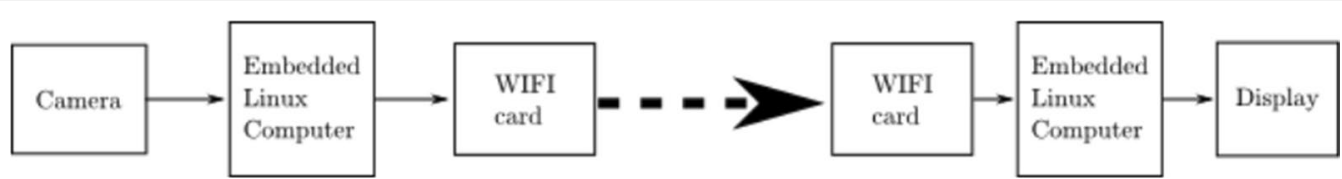

Fig. 2 Schematic plan for the novel wireless endoscope 

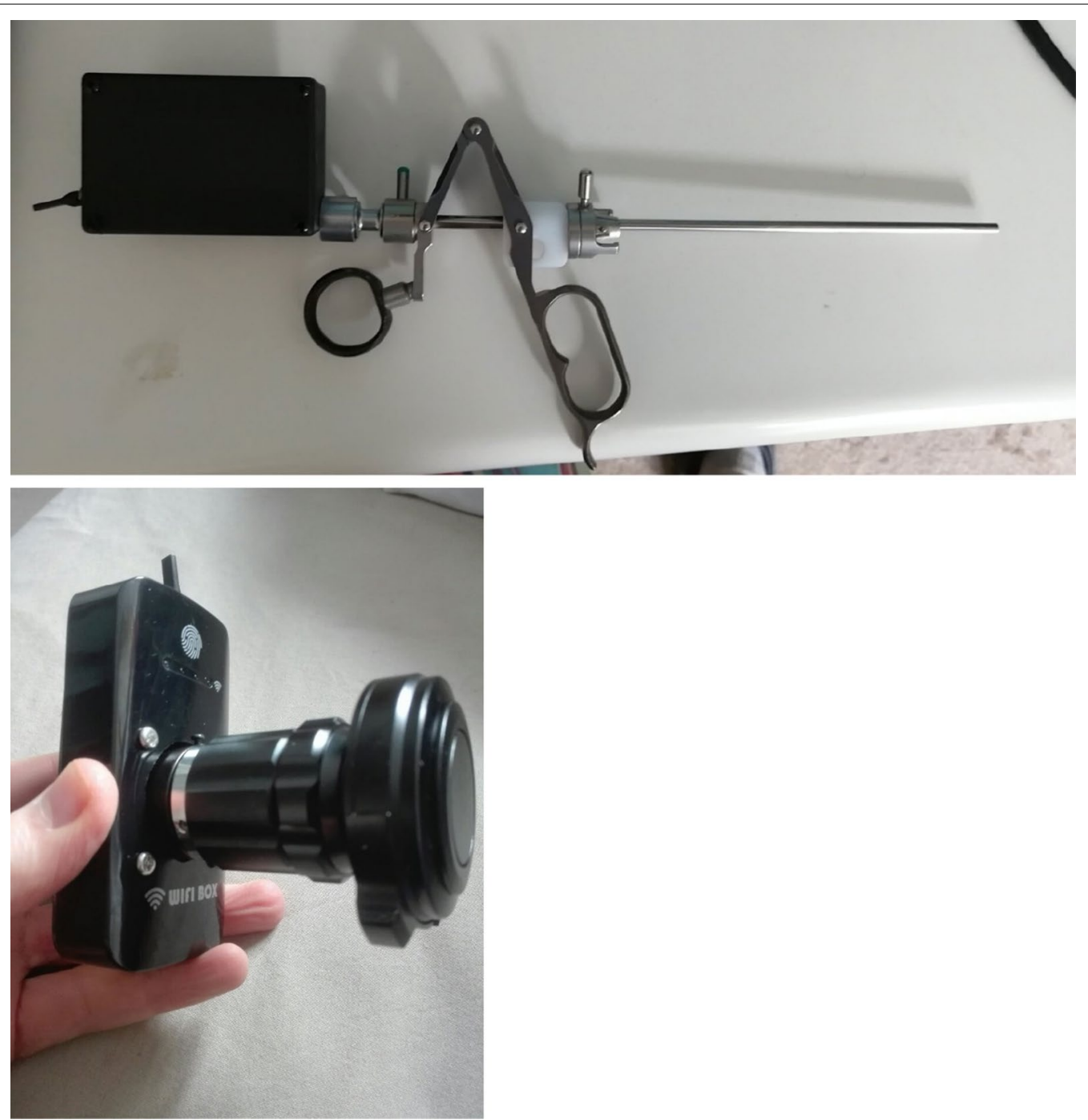

Fig. 3: $4 \mathrm{~mm}$ wireless cystoscope camera attached to a working element of a resectoscope (top). Wireless camera with optical coupler
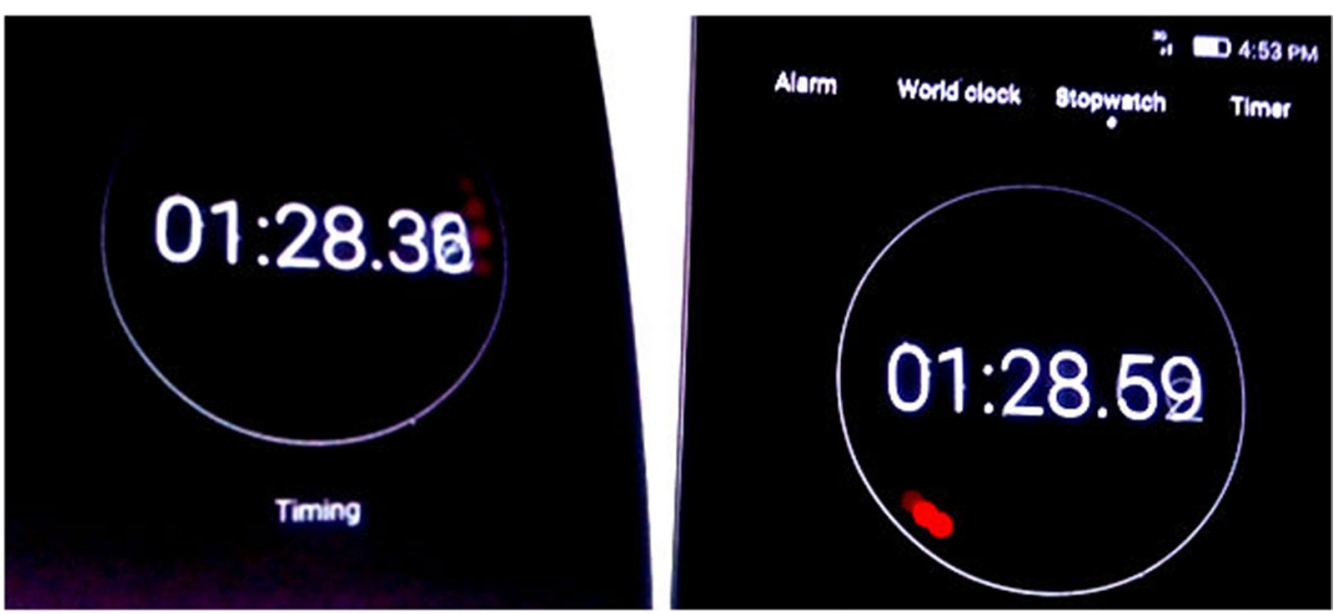

Fig. 4 Near real-time wireless video transmission with a virtually imperceptible latency of $200 \mathrm{~ms}$. Left above is photograph of mobile phone stopwatch, and right image is photograph of computer monitor with wireless transmission from camera 
Traditional Hopkins rod lenses are expensive and fragile. Their replacement by affordable CMOS cameras offers potential savings for healthcare systems. Wireless cameras also offer a significantly simplified system and resultant greater portability compared to wired systems. This portability holds potential for expanded the use of endoscopy outside traditional operating theatres into outpatient setting or even to the bedside.

LED illumination also required no cables or expensive high-powered light sources.

Limitations of this study include the fact that bench testing does not simulate the real-world surgical use. However, we feel the prototype described here offers enough promise to warrant safety, efficacy and acceptability evaluation in a future pilot trial.

\section{Conclusion}

This paper has described the development of an affordable wireless endoscope. Wireless technology has the potential to overcome some of the limitations of traditional wired solutions. We feel the prototype warrants future study in a pilot clinical trial.

\section{Abbreviations}

MIS: minimal access surgery; LED: light emitting diode; SBC: single-board computer.

\section{Acknowledgements}

None.

\section{Author contributions}

$J \mathrm{~L}$ was involved in design and testing of device as well as writing of the manuscript. MN was involved in manufacture of the device and writing of the manuscript. Both authors have read and approved the manuscript.

\section{Funding}

None.

\section{Availability of data and materials}

Data available on request.
Ethical approval and consent to participate

Not applicable-benchtop research, no human subjects.

\section{Consent for Publication}

Not applicable.

\section{Competing interests}

The authors hold no patent over this device and assert no conflict of interest.

Received: 4 August 2020 Accepted: 19 January 2021

Published online: 12 February 2021

\section{References}

1. Bhatt J, Jones A, Foley S, Shah Z, Malone P, Fawcett D, Kumar S (2010) Harold Horace Hopkins: a short biography. BJU Int 106(10):1425-1428

2. Fuchs GJ (2006) Milestones in endoscope design for minimally invasive urologic surgery: the sentinel role of a pioneer. Surg Endosc 20(2):S493-\$499

3. Nezhat's History of Endoscopy, http://laparoscopy.blogs.com/endos copyhistory/. Accessed 11 th July 2020.

4. Endoscope Design and Assembly, https://www.ebme.co.uk/articles/clini cal-engineering/endoscope-design-and-assembly. Accessed 11th July 2020.

5. Chen $\mathrm{CH}$, Chang H, Liu TP, Huang TS, Chen CH (2015) Application of wireless electrical non-fiberoptic endoscope: potential benefit and limitation in endoscopic surgery. Int J Surg 19:6-10

6. Bae JK, Vavilin A, You JS, Kim H, Ryu SY, Jang JH, Jung W (2017) Smartphone-based endoscope system for advanced point-of-care diagnostics: feasibility study. JMIR mHealth uHealth 5(7):e99

7. Li Z, Chiu PW (2018) Robotic endoscopy. Visc Med 34(1):45-51

8. Koulaouzidis A, lakovidis DK, Karargyris A, Rondonotti E (2015) Wireless endoscopy in 2020: Will it still be a capsule? World J Gastroenterol 21(17):5119-5130

9. Operating system images, https://www.raspberrypi.org/downloads/ raspberry-pi-os/. Accessed 11 th July 2020.

10. Raspap-Webgui, https://raspap.com/. Accessed 11th July 2020.

11. (advanced) Projects, https://www.linux-projects.org/uv4l/. Accessed 11th July 2020

\section{Publisher's Note}

Springer Nature remains neutral with regard to jurisdictional claims in published maps and institutional affiliations.

\section{Submit your manuscript to a SpringerOpen ${ }^{\circ}$ journal and benefit from:}

- Convenient online submission

$\checkmark$ Rigorous peer review

- Open access: articles freely available online

- High visibility within the field

- Retaining the copyright to your article

Submit your next manuscript at springeropen.com 